International Journal of Modern Physics E (c) World Scientific Publishing Company

\title{
The Density Matrix Renormalization Group and the Nuclear Shell Model
}

\author{
S. Pittel \\ Bartol Research Institute and Department of Physics and Astronomy, University of Delaware, \\ Newark, DE 19086, USA \\ pittel@bartol.udel.edu \\ B. Thakur \\ Bartol Research Institute and Department of Physics and Astronomy, University of Delaware, \\ Newark, DE 19086, USA \\ N. Sandulescu \\ Institute of Physics and Nuclear Engineering, 76900 Bucharest, Romania \\ Received (received date) \\ Revised (revised date)
}

\begin{abstract}
We summarize recent efforts to develop an angular-momentum-conserving variant of the Density Matrix Renormalization Group method into a practical truncation strategy for large-scale shell model calculations of atomic nuclei. Following a brief description of the key elements of the method, we report the results of test calculations for ${ }^{48} \mathrm{Cr}$ and ${ }^{56} \mathrm{Ni}$. In both cases we consider nucleons limited to the $2 \mathrm{p}-1 \mathrm{f}$ shell and interacting via the KB3 interaction. Both calculations produce a high level of agreement with the exact shell-model results. Furthermore, and most importantly, the fraction of the complete space required to achieve this high level of agreement goes down rapidly as the size of the full space grows.
\end{abstract}

\section{Introduction}

In the nuclear shell model, the low-energy structure of a nucleus is traditionally described by assuming an inert doubly-magic core and diagonalizing the effective nuclear hamiltonian in an active space involving at most a few major shells. Despite the enormous truncation inherent in this approach, the method can still only be applied in very limited nuclear regimes. For sufficiently heavy nuclei, for example, further truncation further is required to reduce the number of shell-model configurations to a manageable size.

An attractive truncation possibility is provided by the Density Matrix Renormalization Group (DMRG), a method initially developed for low-dimensional quantum lattices ${ }^{1}$, and later extended to finite Fermi systems. In the latter context, it has been applied to the description of small metallic grains 2 , to problems in quantum chemistry $\underline{3}$ and to two-dimensional electrons in strong magnetic fields 4 . The 
successes achieved in these various applications suggests that it might also prove useful as a dynamical truncation strategy for obtaining accurate approximate solutions to the nuclear shell model.

The DMRG method involves a systematic inclusion of the degrees of freedom of the many-body problem. When treating quantum lattices, real-space sites are added iteratively. In finite Fermi systems, these sites are replaced by single-particle levels. At each stage, the system [referred to as a block] is enlarged to include an additional site or level. This enlarged block is then coupled to the rest of the system (the medium) giving rise to the superblock. For a given eigenstate of the superblock (often the ground state) or perhaps for a group of important eigenstates, the reduced density matrix of the enlarged block in the presence of the medium is constructed and diagonalized and those states with the largest eigenvalues are retained.

This process of systematically growing the system and determining the optimal structure within that enlarged block is carried out iteratively, by sweeping back and forth through the sites, at each stage using the results from the previous sweep to define the medium. In this way, the process iteratively updates the information on each block until convergence from one sweep to the next is achieved. Finally, the calculations are carried out as a function of the number of states retained in each block, until the changes are acceptably small.

The traditional DMRG method, when applied in nuclei and elsewhere, works in a simple product space, whereby the enlarged block is obtained as a product of states in the block and the added site and likewise the superblock is obtained as a product of states in the enlarged block and the medium. In the context of nuclear terminology, this is equivalent to working in the m-scheme.

A limitation of the traditional algorithm is that it does not preserve symmetries throughout the iterative enlargement process. Since the density matrix procedure involves a truncation at each of the iterative stages, there is a potential to lose these symmetries and the associated correlations. On this basis, we proposed $\frac{5}{5}$ the adoption of a strategy whereby angular momentum is preserved throughout the iterative DMRG process. This method, called the JDMRG, was applied in nuclear physics for the first time in the context of the Gamow Shell Model $\underline{6}$. It was subsequently developed for application to the traditional shell model by Pittel and Sandulescu ${ }^{7}$, where a first test application to ${ }^{48} \mathrm{Cr}$ was reported.

We have now dramatically improved the JDMRG algorithm, to the point where it can be applied to significantly heavier nuclei. In this presentation, we report test results for the largest calculations carried out to date using this method, for the nucleus ${ }^{56} \mathrm{Ni}$.

An outline of the paper is as follows. In section II, we provide a brief overview of the traditional DMRG method including a discussion of the changes required to incorporate angular-momentum conservation throughout. In Section III, we report improved calculations for ${ }^{48} \mathrm{Cr}$ relative to those of ref. 7. Then in Section IV, we report our recently-obtained results for ${ }^{56} \mathrm{Ni}$. Finally, in Section $\mathrm{V}$ we summarize the principal conclusions of this work and outline several directions for future study. 


\section{An overview of the DMRG method}

\subsection{Truncation}

The DMRG method is based on an iterative inclusion of the degrees of freedom of the problem, represented as a chain of sites on a lattice. This is illustrated schematically in Figure 1 for a system with 8 ordered sites.

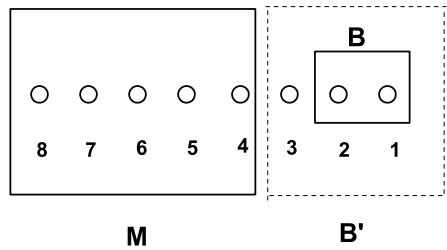

Fig. 1. Schematic illustration of the DMRG growth procedure. A block $B$ consisting of sites 1 and 2 is enlarged to include site 3 , forming $B^{\prime}$. The medium $M$ consists of all of the remaining sites, 4 through 8 .

Assume that we have treated a group of these sites, referred to as the block and denoted $B$, and that we have retained a total number of states $m$ within that block. We now wish to add to this block the next site, which we assume contains $l$ states, thereby producing an enlarged block $B^{\prime}$. For the moment, we will assume a product (or m-scheme) description, so that the enlarged block has $m \times l$ states:

$$
\left|i, j>_{B^{\prime}}=\right| i>_{B} \mid j>_{l} \quad, \quad i=1, m \quad, \quad j=1, l
$$

As typical in Renormalization Group methods, we would like to retain $m$ states for the enlarged block, exactly the same number as before the enlargement. How do we choose them in an optimal way?

In the DMRG method, we consider the enlarged block in the presence of a medium $M$ that reflects all of the other sites of the system, referring to the full system as the superblock (SB). Assuming that the medium is likewise described by $m$ states, the states of the superblock, $m \times l \times m$ in number, can be expressed as

$$
\left|i, j, k>_{S B}=\right| i, j>_{B^{\prime}} \mid k>_{M}
$$

We then diagonalize the full hamiltonian of the system in the superblock, for now isolating on its ground state,

$$
\left|G S>_{S B}=\sum_{i, j, k} \Psi_{i j k}\right| i, j, k>_{S B}
$$

If we then construct the reduced density matrix of the enlarged block in the ground 
state,

$$
\rho_{i j, i^{\prime} j^{\prime}}=\sum_{k} \Psi_{i j k}^{*} \Psi_{i^{\prime} j^{\prime} k}
$$

diagonalize it and retain the $m$ eigenstates with the largest eigenvalues we are guaranteed to have the $m$ most important (or optimal) states of the enlarged block in the ground state (3) of the superblock.

It is straightforward to target a group of states of the system and not just the ground state. To do so, we would construct a mixed density matrix containing information on the block content of all of them.

Once the optimal $m$ states are chosen, we renormalize all required operators of the problem to the truncated space and store this information. This would include all sub-operators of the hamiltonian, viz:

$$
a_{i}^{\dagger}, a_{i}^{\dagger} a_{j}, a_{i}^{\dagger} a_{j}^{\dagger}, a_{i}^{\dagger} a_{j}^{\dagger} a_{k}, a_{i}^{\dagger} a_{j}^{\dagger} a_{k} a_{l}, \quad+\text { h.c. }
$$

Having this information for the block and the additional level or site enables us to calculate all such matrix elements for the enlarged block as needed in the iterative growth procedure.

\subsection{Steps of the DMRG method}

With this as background, the DMRG procedure then involves the following steps.

\subsubsection{Choice of an order for the sites}

Given a hamiltonian and the set of sites in which it is to act, we need to define an order in which the sites are going to be iteratively included. Depending on the number of sites, we might wish to consider many (and perhaps all) orders to see which leads to the energetically lowest solution. It is generally accepted that the optimal order is one in which maximally entangled sites/levels sit near one another ${ }^{8}$.

\subsubsection{The warmup stage}

We begin the iterative process with what is traditionally called the warmup stage. Here, we make an initial guess on the optimal $m$ states for each possible block that will be constructed. This choice will be important in determining how rapidly the iterative procedure will converge. In our treatment, we do this by growing blocks from each side of the chain gradually, using those orbits that have already been treated on the other end as the medium. This is illustrated in Figure 2 for the case of enlargement of the right block from 2 to 3 sites, in the presence of a medium involving the two-site left block already treated. 


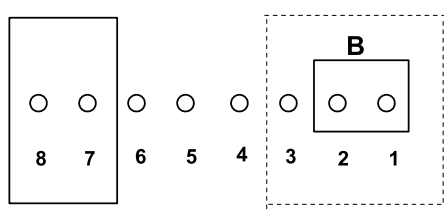

M

B'

Fig. 2. Schematic illustration of the warmup stage as used in the calculations reported herein.

\subsubsection{The Sweep stage}

In this stage of the iterative process, we gradually sweep through the sites of the chain, using for the medium the results either from the warmup phase (during the first sweep) or from the previous sweep stage. As suggested earlier, this sweeping process is done over and over until convergence is achieved in the results from one sweep to the next.

\subsubsection{As a function of $m$}

The warmup and sweep steps just described are typically done for a given choice of $m$. The calculations are then carried out as a function of $m$, until the changes with increasing $m$ are acceptably small.

There is an alternative approach that has been proposed whereby a given set of calculations are carried out not as a function of $m$ but rather as a function of the fraction of the complete density matrix that is retained 9 .

The calculations we will report are based on the former approach.

\subsection{The JDMRG approach}

As noted earlier, most DMRG approaches violate symmetries. In nuclei, for example, they typically work in the m-scheme. When imposing truncation in such a procedure, however, it is difficult to ensure that the states retained contain all the components required by the Clebsch Gordan series to build states of good angular momentum. For this reason, we have chosen to develop an angular-momentumconserving variant of the DMRG method in which angular momentum is preserved throughout the growth, truncation and renormalization stages, referring to it as the JDMRG method.

The JDMRG approach follows the traditional DMRG approach outlined above in most respects. The most significant change is that now we must calculate and store throughout the iterative process the reduced matrix elements of all suboperators of the hamiltonian, namely 
Authors' Names

$$
\begin{gathered}
a_{i}^{\dagger},\left[a_{i}^{\dagger} \tilde{a}_{j}\right]^{K},\left[a_{i}^{\dagger} a_{j}^{\dagger}\right]^{K},\left(\left[a_{i}^{\dagger} a_{j}^{\dagger}\right]^{K} \tilde{a}_{k}\right)^{L}, \\
\left(\left[a_{i}^{\dagger} a_{j}^{\dagger}\right]^{K}\left[\tilde{a}_{k} \tilde{a}_{l}\right]^{K}\right)^{0}+\text { h.c. }
\end{gathered}
$$

This can be done using standard Racah algebra methods.

\subsection{A three-block JDMRG strategy}

In the calculations we will report, we adopt a three-block strategy for the enlargement and truncation process. The basic ideas are summarized in Figure 3.

We begin by choosing our order of sites so that neutron and proton orbitals sit on opposite ends of the chain. We then gradually grow blocks of each type of particle only, namely we grow neutron blocks and proton blocks but no mixed blocks. Lastly, in the sweep stage we go to and fro through the orbits of a given type of particle only. As can be seen from the figure, the medium in this approach involves two components. If, for example, we are enlarging a proton block, as in the figure, the full medium $(M)$ involves all of the remaining proton levels and all of the neutron levels.

This strategy, which was first proposed in ref. 5 , has been found to be computationally more efficient than the two-block approach that is customarily implemented when dealing with systems involving two types of particles in which mixed blocks are constructed.

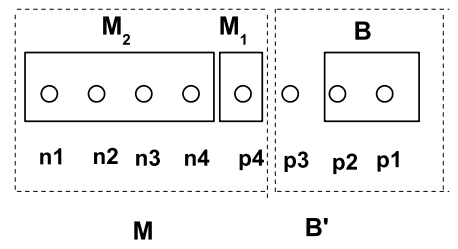

Fig. 3. Schematic illustration of the three-block DMRG growth procedure for a system with neutron and proton levels.

\section{Calculations}

We have carried out test calculations of this three-block JDMRG approach on the nuclei ${ }^{48} \mathrm{Cr}$ and ${ }^{56} \mathrm{Ni}$. As in the usual shell model approach, we assume that these nuclei can be described in terms of valence neutrons and valence protons outside a doubly-magic ${ }^{40} \mathrm{Ca}$ core. We use the shell-model hamiltonian $\mathrm{KB} 3{ }^{10}$, for which 
exact results are available for all low-lying states in ${ }^{48} \mathrm{Cr}$ [11 and for the ground-state binding energy of ${ }^{56} \mathrm{~N}^{12}$.

We report the results separately for these two applications in the following subsections.

\subsection{Results for ${ }^{48} \mathrm{Cr}$}

Here we discuss the results for ${ }^{48} \mathrm{Cr}$, where as noted earlier preliminary results were presented in ref. 7 . The current code, implemented on an Intel Xeon 5355 processor with 16 GB of memory, runs significantly faster than the earlier calculations, allowing us to test more features of the analysis. The size of the full shell-model space for ${ }^{48} \mathrm{Cr}$ is $1,963,461$ states. Of these, 41,355 are $0^{+}$states, 182,421 are $2^{+}$ states, etc.

These calculations are carried out by assuming an ordering of single-particle levels as shown in Figure 4.

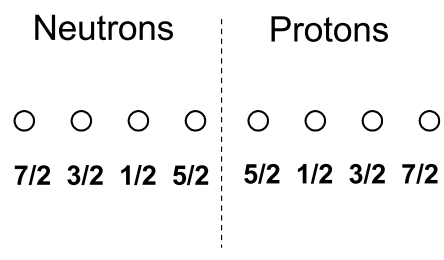

Fig. 4. Order of single-particle levels assumed in all calculations reported herein.

Our results for the ground state are presented in Table 1. The exact calculation produces a ground state energy of $-32.953 \mathrm{MeV}$. The DMRG calculations converge smoothly to this result as $m$ is increased, but require the inclusion of a substantial fraction of the full space to obtain a high level of accuracy. For example, with of order $25 \%$ of the full $0^{+}$space, we can achieve accuracy to only a few $\mathrm{keV}$. Even to achieve an accuracy of $50 \mathrm{keV}$, however, we require roughly $20 \%$ of the full $0^{+}$ space.

It should be noted that the results reported in ref. 7 were for a different order of single-particle levels, explaining why the ground-state energies and maximum dimensions are different for the same $m$ values.

In Table 2, we present the corresponding results for the lowest excited states, obtained for the blocks that derive in the ground state optimization. We only show results for $m \geq 100$. Here too convergence to the exact results is achieved, when a sufficiently large fraction of the full shell-model space is retained, with results of nearly comparable accuracy as for the ground state. This is true despite the fact that the density matrix truncation procedure implemented targeted the ground 


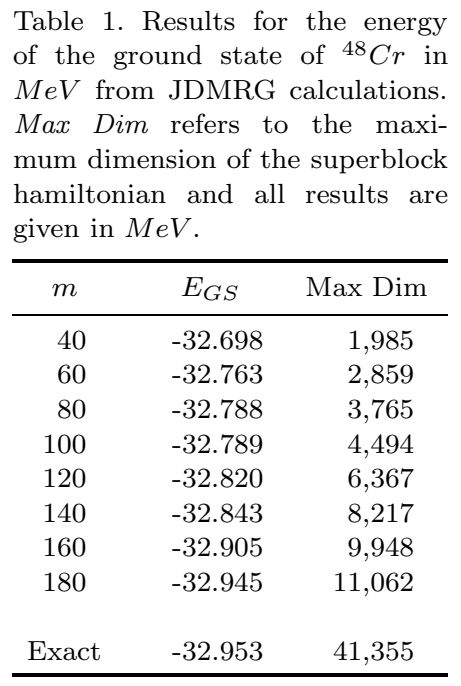

Table 2. Results for the energies of the lowest excited states in $\mathrm{MeV}$ from JDMRG calculations for ${ }^{48} \mathrm{Cr}$. Dim refers to the dimension of the associated superblock hamiltonian and all results are given in $\mathrm{MeV}$.

\begin{tabular}{ccccccccc}
\hline$m$ & $E_{2_{1}^{+}}$ & $(\operatorname{Dim})$ & $E_{4_{1}^{+}}$ & $($Dim $)$ & $E_{6_{1}^{+}}$ & $($Dim $)$ & $E_{0_{2}^{+}}$ & $($Dim $)$ \\
\hline 100 & -31.977 & $(21,003)$ & -30.897 & $(33,261)$ & -29.157 & $(38,652)$ & -27.968 & $(4,494)$ \\
120 & -32.011 & $(28,677)$ & -30.935 & $(42,234)$ & -29.200 & $(45,054)$ & -28.060 & $(6,367)$ \\
140 & -32.040 & $(36,706)$ & -30.979 & $(52,254)$ & -29.264 & $(52,950)$ & -28.152 & $(8,217)$ \\
160 & -32.097 & $(44,454)$ & -31.042 & $(63,222)$ & -29.341 & $(63,298)$ & -28.289 & $(9,948)$ \\
180 & -32.127 & $(50,030)$ & -31.087 & $(72,616)$ & -29.433 & $(74,346)$ & -28.469 & $(11,062)$ \\
& & & & & & & & \\
Exact & -32.148 & $(182,421)$ & -31.130 & $(246,979)$ & -29.555 & $(226,259)$ & -28.564 & $(41,355)$ \\
\hline
\end{tabular}

state only.

\subsection{Results for ${ }^{56} \mathrm{Ni}$}

Next we turn to ${ }^{56} \mathrm{Ni}$, the largest calculation we have performed to date. Now the size of the full shell-model space is significantly larger. The full space in the mscheme contains $1,087,455,228$ states. In an angular momentum basis, the number of $0^{+}$states is $15,443,684$. As noted earlier, we have only considered $0^{+}$states, since those are the only that were calculated in a complete shell-model study with the KB3 interaction. Here too we assumed the order of single-particle levels given in Figure 4.

The results for the ground-state energy as a function of $m$ are shown in table 3 . Here we are able to achieve roughly $60 \mathrm{keV}$ accuracy with barely $1 \%$ of the full $0^{+}$space. 


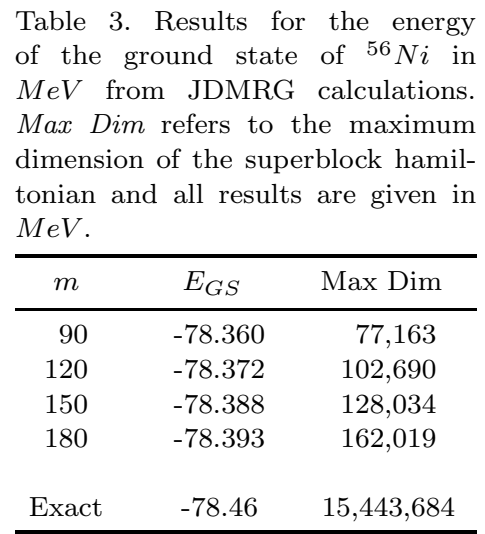

This is perhaps the key result of the study. It suggests that the fraction of the space required to achieve meaningful accuracy with the JDMRG method goes down rapidly as the size of the space increases. If this is confirmed with extension to even larger problems it would bode very well for the future usefulness of the JDMRG method as a practical truncation approach for large-scale shell-model studies.

It should be noted that the DMRG was first applied to ${ }^{56} \mathrm{Ni}$ by Papenbrock and Dean 13 in the traditional m-scheme. Those calculations were unable to obtain the ground state energy to better than $400 \mathrm{keV}$.

As we were completing this study, we learned of a shell-model treatment 14 of ${ }^{56} \mathrm{Ni}$ using the GXPF1A interaction 15 . In that work, complete shell-model results were reported for both the ground-state band and the first deformed band. Clearly, it would be of interest for us to redo our calculations for ${ }^{56} \mathrm{Ni}$ with this interaction, so that we could test the convergence of our method in this nucleus for states other than the ground state.

\section{Summary and Outlook}

In this talk, we have summarized the current status of our efforts to build the Density Matrix Renormalization Group Method method into a practical dynamical truncation strategy for large-scale shell-model calculations of atomic nuclei. Following an overview of the essential features of the method, we discussed the changes we had to implement for its use in application to nuclei. Most importantly, we found it useful to develop an angular-momentum conserving version of the method, the JDMRG. We then summarized the principal results we have obtained to date with this method. We reported test calculations for ${ }^{48} \mathrm{Cr}$ and for ${ }^{56} \mathrm{Ni}$, in both cases comparing with the results of exact diagonalization. Both calculations were able to accurately reproduce the exact shell-model results. In the case of ${ }^{48} \mathrm{Cr}$, however, this high level of accuracy required us to retain a very large fraction of the full space. In contrast, we were able to achieve accurate results for ${ }^{56} \mathrm{Ni}$ with a much 
smaller fraction of the space. The fact that the fraction of the space goes down with the size of the problem is very promising for the future usefulness of the method in even larger shell-model problems.

There are several issues that we intend to explore in the near future. One concerns the need to determine through additional calculations how rapidly the fraction of the space required for convergence scales with the size of the problem. Currently we only have two data points, ${ }^{48} \mathrm{Cr}$ and ${ }^{56} \mathrm{Ni}$. More are needed to draw meaningful conclusions on this key point.

We also have some ideas as to how to break up large single-particle orbitals while still preserving the angular momentum invariance central to the JDMRG. We will test these ideas in the context of the $f_{7 / 2}$ orbital in the current applications and then look into their implementation for even larger orbits.

As noted earlier, we also wish to repeat our test study of ${ }^{56} \mathrm{~N} i$ for the GXPF1A interaction, where exact results also exist for states other than the ground state.

Finally, we will wish to add the calculation of other observables, including those connecting states in different systems.

Once these preliminaries have been completed, we expect to be in a position for many interesting applications of the method to problems of interest in nuclear structure physics.

\section{Acknowledgements}

This work is based on a talk presented by one of the authors (SP) at the International Workshop on Nuclear Structure Physics held in Shanghai, China from 1-7 June 2008. It was supported by the US National Science Foundation under grant \# PHY-0553127. We acknowldege with deep appreciation the important contributions of Jorge Dukelsky to this project. We also wish to thank Alfredo Poves for providing us with the KB3 matrix elements used in this work.

\section{References}

1. S. R. White, Phys. Rev. Lett. 69, 2863 (1992).

2. J. Dukelsky and G. Sierra, Phys. Rev. Lett. 83, 172 (1999); J. Dukelsky and G. Sierra, Phys. Rev. B61, 12302 (2000).

3. S. R. White and R. L. Martin, J. Chem. Phys. 110, 4127 (1999); S. Daul, I. Ciofini, C. Daul, Steven R. White, Int. J. Quantum Chem. 79, 331 (2000).

4. N. Shibata and D. Yoshioka, Phys. Rev. Lett. 86, 5755 (2001); D. Yoshioka and N. Shibata, Physica E12, 43 (2002); N. Shibata, J. Phys. A 36, R381 (2003).

5. J. Dukelsky, and S. Pittel, Rep. Prog. Phys. 67, 513 (2004).

6. N. Michel, W. Nazarewicz, M. Ploszajczak and J. Rotureau, Rev. Mex. Fisica 50, 74 (2004); J. Rotureau, N. Michel, W. Nazarewicz, M. Ploszajczak and J. Dukelsky, Phys. Rev. Lett. 97, 110603 (2006).

7. S. Pittel and N. Sandulescu, Phys. Rev. C 73, 014301 (R) (2006).

8. Ö. Legeza and J. Sólyom, Phys. Rev. B 68, 195116 (2003).

9. Ö. Legeza, J. Röder and B. A. Hess, Phys. Rev. B67, 125114 (2003).

10. A. Poves and A. P. Zuker, Phys. Rep. 70, 235 (1981). 
11. E. Caurier, A. P. Zuker, A. Poves, G. Martínez-Pinedo, Phys. Rev. C 50, 225 (1994).

12. E. Caurier, G. Martínez-Pinedo, F. Nowacki, A. Poves, J. Retamosa and A. P. Zuker, Phys. Rev. C 59, 2033 (1999).

13. T. Papenbrock and D. J. Dean, J. Phys. G 31, S1377 (2005).

14. M. Horoi, B. A. Brown, T. Otsuka, M. Honma and T. Mizusaki, Phys. Rev. C 73, 061305(R) (2006).

15. M. Honma, T. Otsuka, B. A. Brown and T. Mizusaki, Eur. Phys. J. A 25 Supp. 1, 499 (2005). 